\title{
Thermionic emission as a probe of low-energy electron attachment to fullerenes
}

\author{
Bruno Concina, ${ }^{*}$ Franck Lépine, and Christian Bordas \\ Institut Lumière Matière, UMR5306 Université Lyon 1-CNRS, Université de Lyon, 69622 Villeurbanne cedex, France
}

(Received 13 May 2014; published 15 September 2014)

\begin{abstract}
We report on the delayed statistical electron emission of photoexcited fullerene anions $\mathrm{C}_{n}{ }^{-}(n=60,70,78,84$, 90). Kinetic energy release distributions have been measured by a velocity-map imaging spectrometer for delays of about $100 \mathrm{~ns}$ after the nanosecond laser excitation. They show significant variations as a function of the molecular size. Their analysis in the frame of the detailed balance requires the description of the reverse process, namely the electron attachment to the neutral fullerene. For this purpose, the quantum Vogt and Wannier's formalism is used, which allows introducing an empirical expression for the intramolecular vibrational redistribution (IVR). Information on the IVR has been deduced from the fit of the experimental spectra and has been interpreted using the molecular symmetries. In particular $\mathrm{C}_{60}$ has an original behavior related to its icosahedral symmetry.
\end{abstract}

DOI: 10.1103/PhysRevA.90.033415

PACS number(s): 33.80.Eh, 36.40.Qv

Low-energy electron attachment to large molecules such as fullerenes is of great interest but has remained a challenge for both experiment and theory [1-4]. The experimental difficulties were illustrated by the controversy over the attachment to $\mathrm{C}_{60}$ at threshold: Earlier crossed beam measurements [5-7] suggested an activation barrier of about $0.2 \mathrm{eV}$ attributed to the absence of $s$-wave process. Nevertheless, this feature was invalidated by new experiments [8-11].

In general the anion formation may be pictured as a two-step process: electron capture in the polarization potential and transfer of the electron excess energy to the molecular vibrations to lead to a stable anionic state. The first step is described by the classical Langevin model [12] at the most basic level of approximation or by its quantum extension, the Vogt and Wannier model [13]. The second step is called intramolecular vibrational redistribution (IVR) or electronphonon coupling and is expected to depend on the ionic core symmetries by some selection rules [3]. Fullerenes are ideal to shed light on these selection rules as they present a large range of symmetries from the icosahedral one for $\mathrm{C}_{60}$, and the $D_{5 h}$ one for $\mathrm{C}_{70}$, to much lower symmetries for the larger fullerenes.

As a consequence of the detailed-balance principle, the thermionic (statistical) emission of the fullerene anions is related to the reverse process, namely the electron attachment to the neutral fullerenes [14]. In this frame, the kinetic energy release distribution (KERD) of the emitted electrons depends on the attachment cross section. In this paper, we report on the measurement of the KERD of thermionic emission with a velocity-map imaging spectrometer. The KERD has been analyzed in the frame of the detailed balance using the quantum Vogt and Wannier model [13] for the attachment cross section. This model is well suited for the empirical description of the molecular aspects of the attachment such as IVR [15]. Strong variations of the KERD are observed as a function of the fullerene size and are proposed to be related to the molecular symmetries.

Let us point out the advantages of our approach in the study of the electron attachment at threshold. In the low-energy $(<0.3 \mathrm{eV})$ crossed beam experiments, the electron current

\footnotetext{
*bruno.concina@univ-lyon1.fr
}

strongly decreases in a poorly known way which prevents one from measuring cross sections $[4,10]$. The reported resolution lies between 30 and $150 \mathrm{meV}$ and the zero of the energy scale must be checked with a molecule known to have a $0-\mathrm{eV}$ resonance (e.g., $\mathrm{SF}_{6}$ ) [4,10]. By comparison the velocity-map imaging spectrometer is well suited for threshold measurements: constant detection efficiency and a resolution that gets higher when the kinetic energy decreases (see below). The zero of the energy scale is accurately determined as the center of the raw image.

The experimental setup will be described in detail elsewhere. Briefly, the isolated fullerene anions are produced by electrospray ionization (ESI). A tetrakis(dimethylamino)ethylene (TDAE) solution (1 $\mathrm{mM}$ in dichloromethane) is added to the fullerene solution $(0.1 \mathrm{mM}$ in toluene) in the ratio 7:3. Addition of the electron donor TDAE was shown to improve production of isolated fullerene anions by several orders of magnitude [16]. The ESI source is part of a commercial mass spectrometer, micrOTOF-Q (Bruker Daltonics). The selection of the ionic species of interest is operated with a mass resolving quadrupole. At the exit of the micrOTOF-Q, the ions are perpendicularly accelerated to a linear time of flight (TOF) by a Wiley-McLaren assembly with high voltages pulsed at a $350-\mathrm{Hz}$ repetition rate. The linear TOF contains the velocity-map imaging (VMI) spectrometer mounted orthogonally to the ion drift tube. The photoelectron imaging system is based on the design introduced by Eppink and Parker [17] and is very like the one described in Ref. [18]. Briefly, an inhomogeneous electric field perpendicular to both the ion trajectory and the focused laser beam accelerates the electrons towards a position-sensitive detector (a microchannel plate detector backed by a phosphor screen). Electron impacts are recorded by a charge coupled device (CCD) camera. The angular-dependent velocity distribution is deduced from the raw image through numerical inversion [19]. It is integrated over angles to get the electron KERD. The nanosecond pulsed detachment lasers operating at a $350-\mathrm{Hz}$ repetition rate are a diode pumped Nd:YAG laser (1064 nm), eventually frequencydoubled $(532 \mathrm{~nm})$, and a diode pumped frequency-tripled $\mathrm{Nd}$ :YLF laser (349 $\mathrm{nm})$. The polarization is linear and parallel to the position-sensitive detector surface. The time resolution of the VMI is achieved by gating the voltages applied on the position-sensitive detector, making the detection efficient 


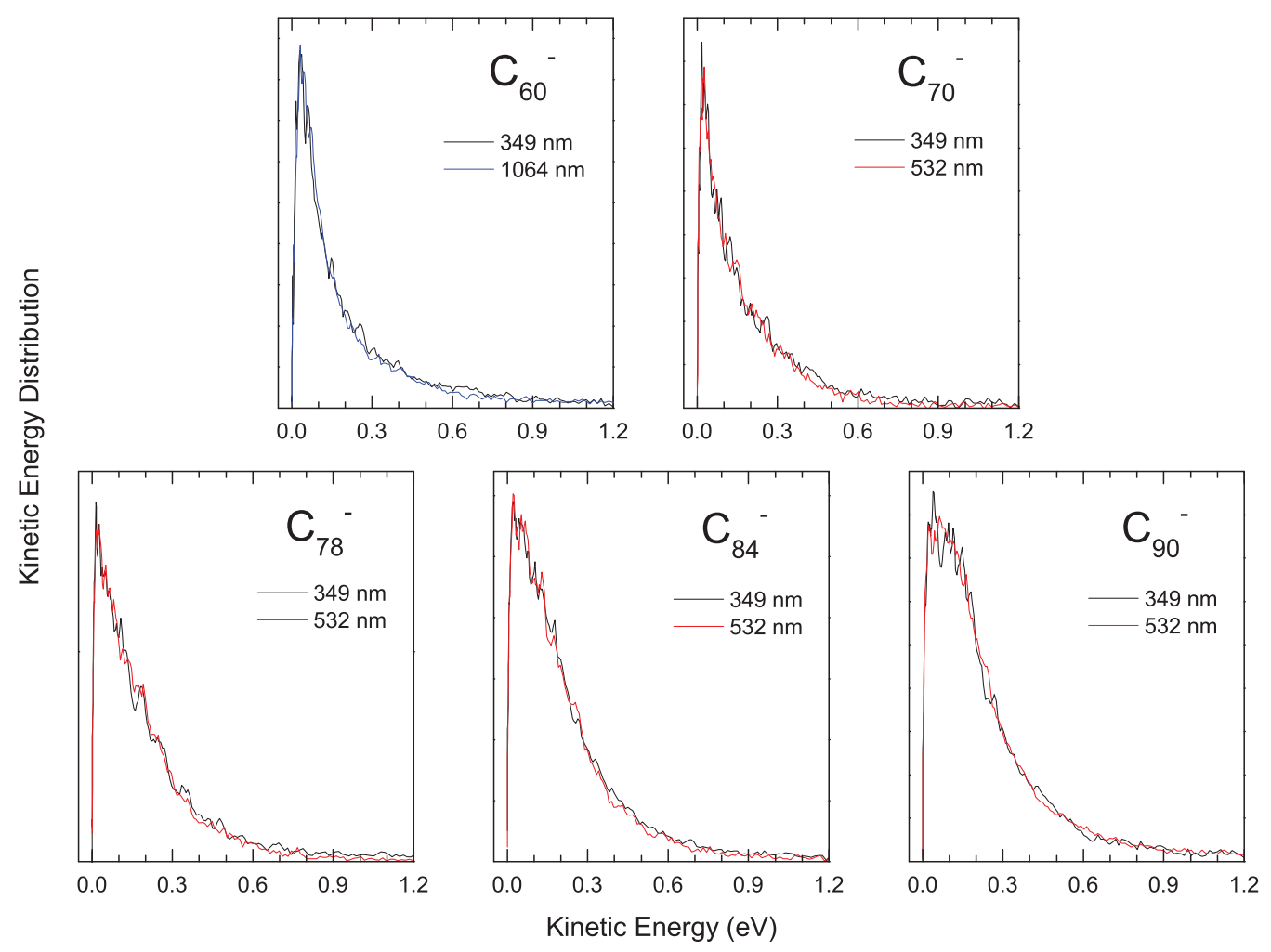

FIG. 1. (Color online) Experimental kinetic energy release distributions of delayed electron emission from mass-selected fullerene anions $\mathrm{C}_{n}{ }^{-}(n=60,70,78,84,90)$ at three laser wavelengths $(349,532$, and $1064 \mathrm{~nm})$.

during approximately $35 \mathrm{~ns}$ [full width at half maximum (FWHM) of the detection efficiency curve]. The imaging spectrometer has been calibrated with the spectrum of $\mathrm{I}^{-}$at $349 \mathrm{~nm}$ and a kinetic energy resolution $\Delta \varepsilon$ of $85 \mathrm{meV}$ (FWHM) has been measured at $\varepsilon=0.494 \mathrm{eV}$. Assuming that the velocity resolution $\Delta v$ is constant, $\Delta \varepsilon$ is found proportional to $\sqrt{\varepsilon}$, leading to $\Delta \varepsilon=40 \mathrm{meV}$ at $\varepsilon=0.1 \mathrm{eV}$ and even less at lower energy, which compares well with the resolution of the attachment experiments.

The detection window is delayed by $110 \mathrm{~ns}$ with respect to the laser pulse for the $349 \mathrm{~nm}$ measurements and by $150 \mathrm{~ns}$ for the 532 and $1064 \mathrm{~nm}$ spectra. For the three laser wavelengths, no contribution from direct photodetachment is expected, as its time scale is much shorter than a nanosecond. Consequently, pure delayed electron emission spectra for well-defined time delays are recorded. Measurement of the delayed electron emission from the mass-selected fullerene anions $\mathrm{C}_{n}{ }^{-}(n=$ $60,70,78,84,90)$ has been attempted with the three laser wavelengths and a sufficient photoelectron rate has been observed for the cases reported in Fig. 1. For the other cases, very low photoelectron rates have been measured which may be caused by a low absorption cross section at play in this multiphoton process [20]. The two spectra for a given size are identical, confirming the thermionic (statistical) nature of the measured electron emission. On the contrary, the KERD profile depends on the fullerene size. The spectrum is quite narrow $\left(\sim 100 \mathrm{meV}\right.$ ) for $\mathrm{C}_{60}{ }^{-}$and $\mathrm{C}_{70}{ }^{-}$and it becomes broader when the size increases $\left(\sim 250 \mathrm{meV}\right.$ for $\left.\mathrm{C}_{90}{ }^{-}\right)$. Such a behavior is rationalized using the detailed-balance theory and the quantum capture cross section, as detailed below.
The description of thermionic emission is based on the assumption of a thermal equilibrium among all vibrational degrees of freedom. It relies on the microscopic reversibility, also called the detailed-balance principle. Following the Weisskopf model [21], the KERD depends on the cross section $\sigma(\varepsilon)$ of the reverse process, namely the electron attachment to the neutral fullerene [14,22,23]:

$$
f(\varepsilon) \propto \varepsilon \sigma(\varepsilon) \exp \left(-\frac{\varepsilon}{k_{B} T_{d}}\right),
$$

with $T_{d}$ the microcanonical temperature of the daughter (i.e., the neutral fullerene after electron emission). The crucial quantity is the attachment cross section $\sigma(\varepsilon)$. A first estimate is provided by the Langevin cross section $\sigma_{L}(\varepsilon)$ which classically describes the capture in the polarization potential $V(r)=$ $-c_{4} / r^{4}$ with $c_{4}=\alpha\left(e^{2} / 4 \pi \varepsilon_{0}\right) / 2, \alpha$ being the polarizability of the neutral fullerene, and which reads $\sigma_{L}(\varepsilon)=2 \pi \sqrt{c_{4} / \varepsilon}$ [12]. The KERD assuming the Langevin cross section (with $T_{d}$ as a free parameter) cannot fit the sharp peaks observed in several experimental distributions $\left(\mathrm{C}_{60}{ }^{-}\right.$and $\mathrm{C}_{70}{ }^{-}$, for example). This implies that a more realistic description of the electron attachment to the neutral fullerene including IVR should be used. We propose to introduce it using the quantum formalism of Vogt and Wannier.

The quantum problem of the capture in the polarization potential was solved in the 1950s by Vogt and Wannier [13]. The quantum cross section oscillates about the Langevin estimate except at low energies, where it tends to twice this classical limit [13]. In the case of $\mathrm{C}_{60}$, it was shown that the difference between the Langevin and the Vogt and Wannier 
cross sections does not exceed $5 \%$ for energies above $4 \mathrm{meV}$ [1]. In the quantum approach, the capture cross section $\sigma$ is the sum of the contributions of the partial waves of given orbital momentum $\ell$ :

$$
\sigma(k)=\sum_{\ell} \frac{\pi}{k^{2}}(2 \ell+1) P_{\ell}^{\mathrm{VW}}(k),
$$

$k$ being the wave vector and $P_{\ell}^{\mathrm{VW}}(k)$ the orbital momentumdependent capture probability. The angular dependence of the wave functions is given by the spherical harmonics. The $P_{\ell}^{\mathrm{VW}}(k)$ can be written as a function of the reduced wave vector $\kappa=k \sqrt{2 m_{e} c_{4} / \hbar^{2}}$ which simply reads $\kappa=(2 \alpha \varepsilon)^{1 / 2}$ in atomic units [24]. Using the reduced wave vector, Eq. (2) becomes in atomic units,

$$
\sigma(\kappa)=\alpha \frac{\pi}{\kappa^{2}} \sum_{\ell}(2 \ell+1) P_{\ell}^{\mathrm{VW}}(\kappa) .
$$

Numerically accurate analytical fittings for the $P_{\ell}^{\mathrm{VW}}(\kappa)$ are available [25]. The IVR is introduced via the empirical probabilities $P_{\ell}^{\mathrm{IVR}}(\kappa)$ that an electron captured by the polarization potential leads to a stable anion:

$$
\sigma(\kappa)=\alpha \frac{\pi}{\kappa^{2}} \sum_{\ell}(2 \ell+1) P_{\ell}^{\mathrm{VW}}(\kappa) P_{\ell}^{\mathrm{IVR}}(\kappa) .
$$

We have made some amendments to the $P_{\ell}^{\mathrm{IVR}}(\kappa)$ expressions available in the literature $[15,26]$. For the $s$ wave, we propose an energy-independent probability $P_{0}^{\mathrm{IVR}}(\kappa)=a_{0}$. The extension to higher orbital momenta takes into account

the centrifugal barrier $\kappa_{0}(\ell)=\ell(\ell+1) / 2$ of the fullereneelectron orbiting potential:

$P_{\ell}^{\mathrm{IVR}}(\kappa)=a_{\ell}$ for $\kappa<\kappa_{0}(\ell)$,

$P_{\ell}^{\mathrm{IVR}}(\kappa)=a_{\ell} \exp \left\{-b_{\ell}\left[\kappa^{2}-\kappa_{0}^{2}(\ell)\right]\right\}$ for $\kappa \geqslant \kappa_{0}(\ell)$.

The empirical formalism described above is useful for comparison to the experiment. A rigorous treatment, such as $R$-matrix theory, will not separate electronic and nuclear coordinates $[15,25]$. Let us point out also that the conservation of the angular momentum at play in the attachment process must include the rotation of the fullerene cage. Finally, the $\kappa$ distribution used to fit the experimental ones is deduced from both Eq. (1) and Eq. (4):

$$
f(\kappa) \propto \kappa \exp \left(-\frac{\kappa^{2}}{2 \alpha k_{B} T_{d}}\right) \sum_{\ell}(2 \ell+1) P_{\ell}^{\mathrm{VW}}(\kappa) P_{\ell}^{\mathrm{IVR}}(\kappa),
$$

with $\alpha$ in atomic units, $T_{d}$ in Kelvin, and $k_{B}=3.1668 \times$ $10^{-6} \mathrm{HK}^{-1}$. A benchmark for the daughter temperature is given by a detailed-balance model described in detail in Ref. [14]. Briefly, the decay rate assuming a Langevin cross section is derived and is equaled to the inverse of the delay of the detection window. The temperature depends very little on the profile of the cross section. The calculations require the electron affinity for which we use measurements [27-30] and the polarizability taken from measurements for $\mathrm{C}_{60}$ and $\mathrm{C}_{70}[31,32]$ and scaled by $n^{3 / 2}$ for the larger fullerenes in agreement with the molecular cage structure. A caloric curve
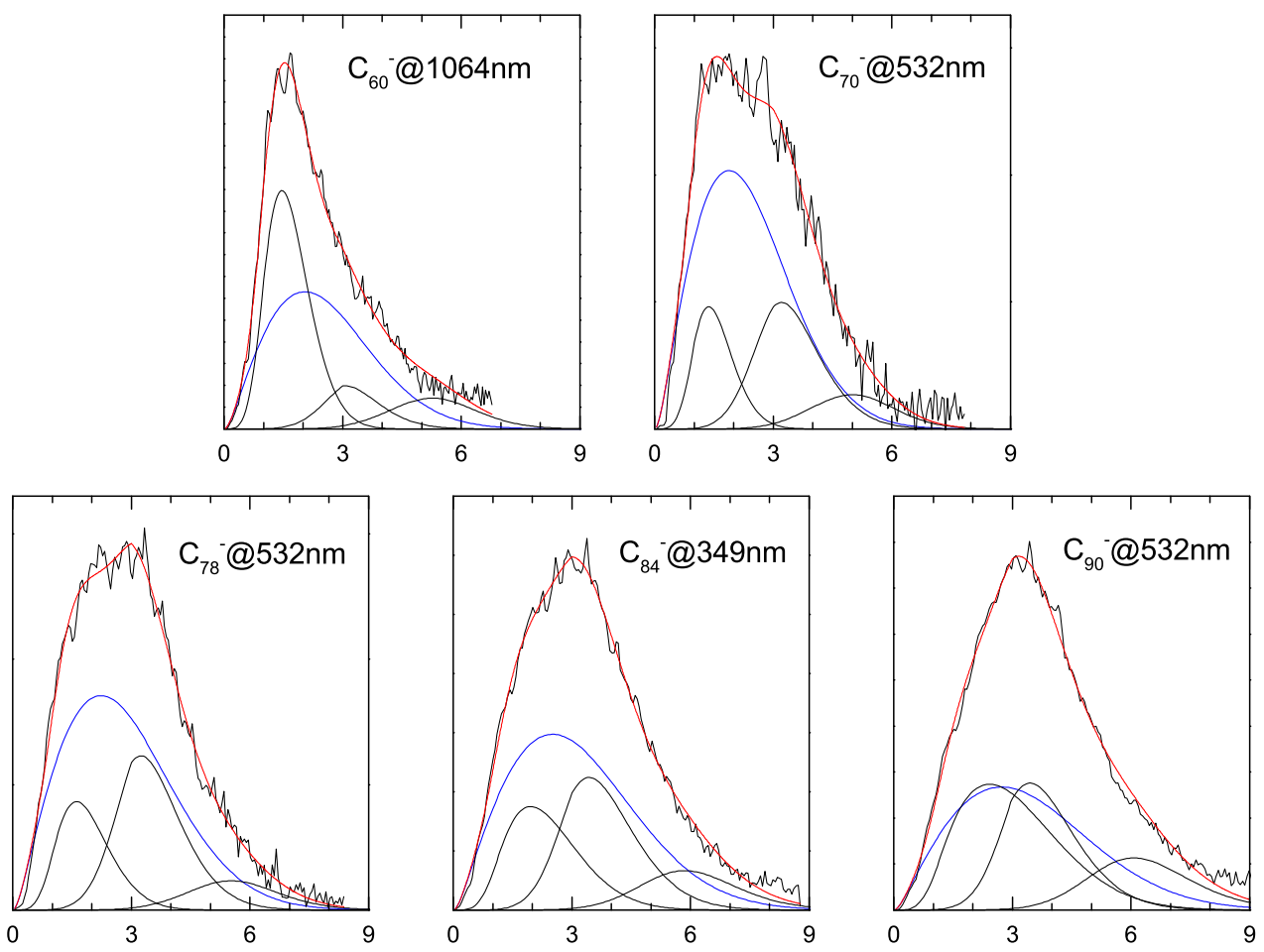

Reduced wave vector $\kappa$

FIG. 2. (Color online) Experimental $\kappa$ distributions (black) compared to the global fits (red) for the delayed electron emission from the fullerene anions $\mathrm{C}_{n}{ }^{-}(n=60,70,78,84,90)$. The contributions of the partial waves $\ell=0,1,2,3$ are also represented (the $s$ wave in blue and the others in black with increasing threshold when $\ell$ increases). 
is also needed which is taken from the literature for $\mathrm{C}_{60}$ [33] and scaled accordingly to $n$ for the larger fullerenes.

The experimental $\kappa$ distributions are derived from the measured velocity distributions. The resolution $\Delta \kappa$ slightly depends on the polarizability and lies between 0.37 for $\mathrm{C}_{60}{ }^{-}$ and 0.51 for $\mathrm{C}_{90}{ }^{-}$. As only the relative weight between the partial waves can be deduced from the fit, the $s$ wave is used as a benchmark and the ratios $\rho_{\ell}=a_{\ell} / a_{0}$ are extracted instead of $a_{\ell}$. In accordance with the $\kappa$ upper limit on the experimental distributions, only the first four partial waves $(\ell \leqslant 3)$ are considered (the $g$-wave threshold is at about $\kappa=7$ ) and the IVR factor $b_{3}$ (which plays a role only for $\kappa>6$ ) is set to zero. As a first approach, a global scale factor, the daughter temperature, and the IVR factors $\rho_{\ell}(\ell=1,2,3)$ and $b_{\ell}(\ell=1,2)$ are assumed to be free in the fitting of the experimental spectra. Nevertheless this leads to a ratio $\rho_{3}(f$ wave) equal to zero and a daughter temperature very different from the model prediction. If the ratio $\rho_{3}$ is set to a given value, the resulting fit cannot be distinguished from the free $\rho_{3}$ fit except at high $\kappa$ where the background signal is not negligible, making it as reliable as the free fit. As a consequence, it is not possible to determine both the temperature and the full shape of the cross section from the fit. Thus the choice of the $\rho_{3}$ value is guided by the agreement of the daughter temperature with the detailed-balance model. The fixed $\rho_{3}$ fit leads to $\rho_{2} \approx \rho_{1}$ for $\mathrm{C}_{78}{ }^{-}, \mathrm{C}_{84}{ }^{-}$, and $\mathrm{C}_{90}{ }^{-}$so for these fullerenes we assume $\rho_{3}=\rho_{2}$. This choice will be interpreted later. It does not lead to reasonable fit parameters for $\mathrm{C}_{60}{ }^{-}$and $\mathrm{C}_{70}{ }^{-}$and so for these anions, we fix $\rho_{3}=1$. An experimental $\kappa$ distribution for each fullerene mass is represented in Fig. 2 with the global fit and the contribution of each partial wave. For all fullerene sizes and consequently for all shapes of $\kappa$ distribution, the fits are in excellent agreement with the experimental distributions. The fit parameters are given as a function of the fullerene size in Fig. 3. The reported parameters correspond to the average of the fit parameters of the two measurements available for each fullerene size. The reported error bars result from the dispersion between the two sets of fit parameters and their respective standard errors. The choice of the $\rho_{3}$ value has led to temperatures in nice agreement with the benchmark, except for $\mathrm{C}_{60}$. A comparison with the attachment experiments necessitates giving the fullerene temperature in both cases. $T_{d}$ can be compared to the temperature of the oven producing the neutral fullerenes, i.e., about $700 \mathrm{~K}$ [10].The larger content of vibrational energy in our experiment may have an impact on the IVR process.

In a simple picture, the IVR depends on three physical ingredients and their respective symmetries: the wave function of the incoming electron, the molecular vibrations, and the lowest unoccupied molecular orbital (LUMO) of the neutral fullerene. The Vogt and Wannier's partial waves describing the incoming electron have the radial symmetry of the spherical harmonics and thus allow probing different symmetries: isotropic for the $s$ wave, two lobes for the $p$ waves, four lobes for the $d$ waves, and so on. The symmetries of the vibrations and of the LUMO derive from the ones of the ionic core. Very different levels of symmetries are observed for the studied fullerenes. Let us recall that $\mathrm{C}_{60}$ belongs to the icosahedral point group $I_{h}$ and $C_{70}$ to the $D_{5 h}$ point group. The larger fullerenes are observed in several isomeric forms of lower
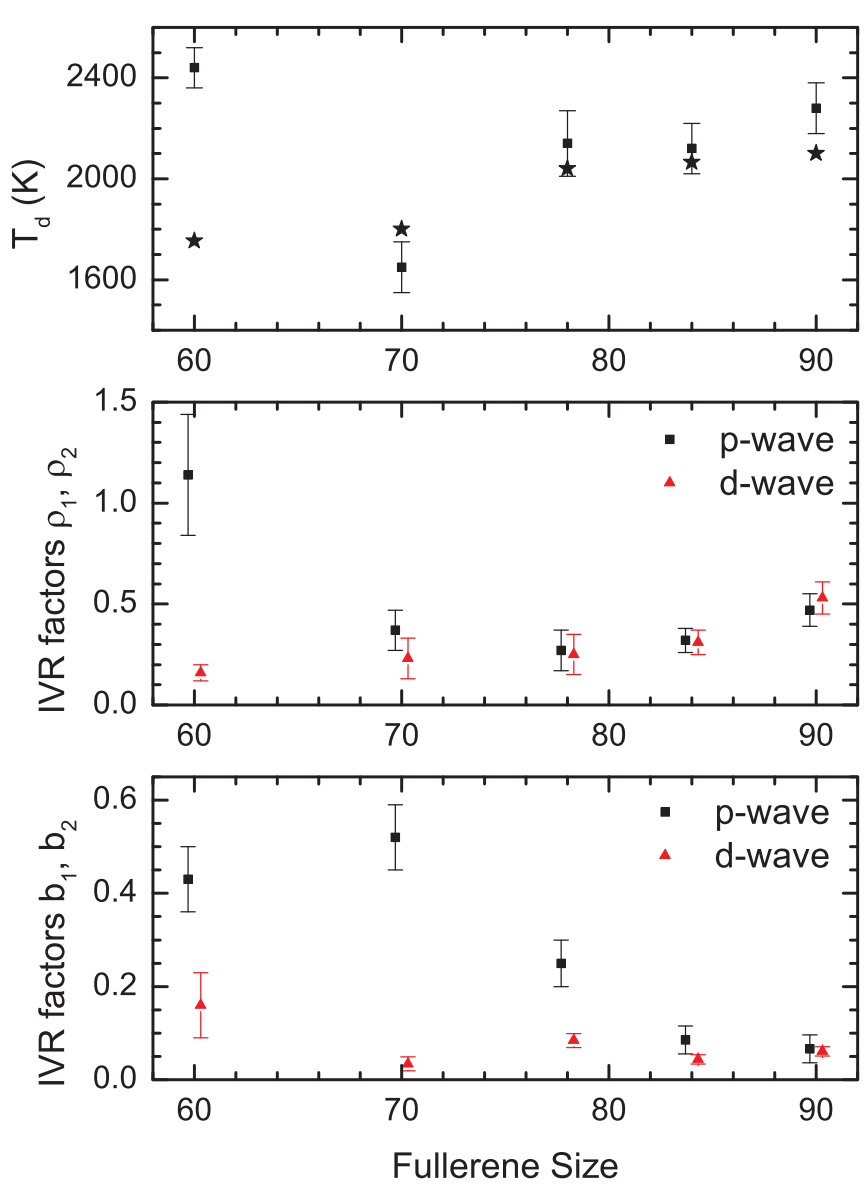

FIG. 3. (Color online) Parameters deduced from the fit of the experimental $\kappa$ distributions as a function of the fullerene size. The benchmark for the daughter temperature (derived from a detailedbalance model) is also represented (black star).

symmetry. $\mathrm{C}_{78}$ consists of three isomers of symmetry $C_{2 v}^{\prime}$, $C_{2 v}$, and $\mathrm{D}_{3}$ in the ratio 5:2:2 [34]. Two isomers $D_{2}$ and $D_{2 d}$ are observed for $\mathrm{C}_{84}$ in the ratio 2:1 [34]. $\mathrm{C}_{90}$ has five isomeric forms: one with $C_{2 v}$ symmetry, three with $C_{2}$ symmetry, and one with $C_{1}$ symmetry [35].

The IVR is characterized by the two orbital momentumdependent parameters $\rho_{\ell}$ and $b_{\ell}$. The ratio $\rho_{\ell}$ is the probability normalized to the isotropic case ( $s$ wave) that a captured electron leads to a stable anionic state if its kinetic energy is below the centrifugal barrier. $b_{\ell}$ describes the exponential decreasing of this probability above the centrifugal barrier. For the molecular sizes between $\mathrm{C}_{70}$ and $\mathrm{C}_{90}$ the IVR probability is lower for the $p$ and $d$ waves in comparison to the isotropic case and it increases when the size goes up. Indeed $\rho_{1}$ and $\rho_{2}$ are almost equal and they both slightly increase. On the contrary, $b_{1}$ and $b_{2}$ behave very differently: $b_{2}$ is quite low and almost constant whereas $b_{1}$ decreases strongly and tends to equal $b_{2}$ for the larger fullerenes. Such size behavior of the IVR is attributed to the strong lowering of the molecular symmetry from $\mathrm{C}_{70}$ to $\mathrm{C}_{90}$. For the larger fullerenes, $\mathrm{C}_{78}, \mathrm{C}_{84}$, and $\mathrm{C}_{90}$, it has been assumed that $\rho_{3}=\rho_{2}$. Considering that we measure $\rho_{2} \approx \rho_{1}$, it is reasonable to admit that there is no selection rule on $\rho_{\ell}$ as a function of $\ell$ for these low-symmetry molecules. 
By contrast with the other molecules, $\mathrm{C}_{60}$ is characterized by a quite high value for $\rho_{1}$ (around or higher than 1) which can be related to its high icosahedral symmetry. Indeed in the $I_{h}$ symmetry, the $p$ waves transform as $t_{1 u}$ which is the symmetry of the LUMO [36]. The fact that the initial and final wave functions have the same symmetry may strengthen the electron-phonon coupling. $b_{1}$ and $b_{2}$ are also both high $\left(b_{1}=0.43\right.$ and $\left.b_{2}=0.16\right)$ and can be compared to the literature. In Ref. [15], Viggiano et al. measured thermal attachment rate to $\mathrm{C}_{60}$. These measurements and experimental attachment cross sections from the literature are compared with theoretical capture cross sections based on the Vogt-Wannier approach including IVR probabilities. Within the rather large experimental uncertainty, they deduced an IVR factor $b$ independent of the orbital momentum $\ell$ : $b \approx 0.2$. Satisfactory agreement is found with our $\ell$-dependent estimates showing nice convergence between attachment and detachment studies. In Ref. [3], Kasperovich et al. measured the electron attachment cross section to $\mathrm{C}_{60}$ at low energy by beam-depletion spectroscopy. They found a deep minimum around $0.4 \mathrm{eV}$. This feature can be linked to the low value of $\rho_{2}$ (in comparison to $\rho_{1}$ ), the $d$ wave reaching its maximum at $0.32 \mathrm{eV}(\kappa=3.5)$.

In conclusion, KERD of the delayed statistical electron emission of fullerene anions has been measured with a velocity-map imaging spectrometer. The analysis of the experimental spectra in the frame of the detailed balance is based on the description of the electron attachment, and more specifically on the empirical description of the IVR. The suitability of the VMI for threshold measurements allows showing a molecular symmetry dependence of the IVR. The increasing of the IVR probability for the $p$ and $d$ waves relatively to the $s$ wave from $\mathrm{C}_{70}$ to $\mathrm{C}_{90}$ is associated to the lowering of the molecular symmetry. Concerning the highly symmetrical $\mathrm{C}_{60}$ a quite high IVR probability is observed for the $p$ wave, which transforms as the LUMO in the icosahedral geometry. This is an original feature for this remarkable molecule.

Financial support of the French "Agence Nationale de la Recherche" (ANR) through Project No. ANR-06-BLAN-0041 is acknowledged. We thank E. Papalazarou for his contribution to the construction of the experimental setup.
[1] H. Hotop, M.-W. Ruf, M. Allan, and I. I. Fabrikant, Adv. At. Mol. Opt. Phys. 49, 85 (2003).

[2] I. I. Fabrikant and H. Hotop, Phys. Rev. A 63, 022706 (2001).

[3] V. Kasperovich, G. Tikhonov, and V. V. Kresin, Chem. Phys. Lett. 337, 55 (2001).

[4] S. Ptasińska, O. Echt, S. Denifl, M. Stano, P. Sulzer, F. Zappa, A. Stamatovic, P. Scheier, and T. D. Märk, J. Phys. Chem. A 110, 8451 (2006).

[5] T. Jaffke, E. Illenberger, M. Lezius, S. Matejcik, D. Smith, and T. D. Märk, Chem. Phys. Lett. 226, 213 (1994).

[6] S. Matejčik, T. D. Märk, P. Španěl, D. Smith, T. Jaffke, and E. Illenberger, J. Chem. Phys. 102, 2516 (1995).

[7] J. Huang, H. S. Carman, and R. N. Compton, J. Phys. Chem. 99, 1719 (1995).

[8] C. D. Finch, R. A. Popple, P. Nordlander, and F. B. Dunning, Chem. Phys. Lett. 244, 345 (1995).

[9] J. M. Weber, M.-W. Ruf, and H. Hotop, Z. Phys. D 37, 351 (1996).

[10] O. Elhamidi, J. Pommier, and R. Abouaf, J. Phys. B: At., Mol. Opt. Phys. 30, 4633 (1997).

[11] Y. V. Vasil'ev, R. F. Tuktarov, and V. A. Mazunov, Rapid Commun. Mass Spectrom. 11, 757 (1997).

[12] P. Langevin, Ann. Chim. Phys. 5, 245 (1905).

[13] E. Vogt and G. H. Wannier, Phys. Rev. 95, 1190 (1954).

[14] B. Concina, B. Baguenard, F. Calvo, and C. Bordas, J. Chem. Phys. 132, 104307 (2010).

[15] A. A. Viggiano, J. F. Friedman, N. S. Shuman, T. M. Miller, L. C. Schaffer, and J. Troe, J. Chem. Phys. 132, 194307 (2010).

[16] O. Hampe, M. Neumaier, M. N. Blom, and M. M. Kappes, Chem. Phys. Lett. 354, 303 (2002).

[17] A. T. J. B. Eppink and D. H. Parker, Rev. Sci. Instrum. 68, 3477 (1997).

[18] B. Baguenard, J. B. Wills, F. Pagliarulo, F. Lépine, B. Climen, M. Barbaire, C. Clavier, M. A. Lebeault, and C. Bordas, Rev. Sci. Instrum. 75, 324 (2004).
[19] C. Bordas, F. Paulig, H. Helm, and D. L. Huestis, Rev. Sci. Instrum. 67, 2257 (1996).

[20] B. Concina, M. Neumaier, O. Hampe, and M. M. Kappes, Int. J. Mass Spectrom. 252, 110 (2006).

[21] V. Weisskopf, Phys. Rev. 52, 295 (1937).

[22] J. U. Andersen, E. Bonderup, and K. Hansen, J. Phys. B 35, R1 (2002).

[23] F. Lépine and C. Bordas, Phys. Rev. A 69, 053201 (2004).

[24] E. I. Dashevskaya, A. I. Maergoiz, J. Troe, I. Litvin, and E. E. Nikitin, J. Chem. Phys. 118, 7313 (2003).

[25] E. E. Nikitin and J. Troe, Phys. Chem. Chem. Phys. 12, 9011 (2010).

[26] J. Troe, T. M. Miller, and A. A. Viggiano, J. Chem. Phys. 130, 244303 (2009).

[27] X.-B. Wang, H.-K. Woo, and L.-S. Wang, J. Chem. Phys. 123, 051106 (2005).

[28] X.-B. Wang, H.-K. Woo, X. Huang, M. M. Kappes, and L.-S. Wang, Phys. Rev. Lett. 96, 143002 (2006).

[29] X.-B. Wang, H.-K. Woo, J. Yang, M. M. Kappes, and L.-S. Wang, J. Phys. Chem. C 111, 17684 (2007).

[30] O. V. Boltalina, E. V. Dashkova, and L. N. Sidorov, Chem. Phys. Lett. 256, 253 (1996).

[31] R. Antoine, P. Dugourd, D. Rayane, E. Benichou, M. Broyer, F. Chandezon, and C. Guet, J. Chem. Phys. 110, 9771 (1999).

[32] I. Compagnon, R. Antoine, M. Broyer, P. Dugourd, J. Lermé, and D. Rayane, Phys. Rev. A 64, 025201 (2001).

[33] J. U. Andersen, C. Gottrup, K. Hansen, P. Hvelplund, and M. O. Larsson, Eur. Phys. J. D 17, 189 (2001).

[34] K. Kikuchi, N. Nakahara, T. Wakabayashi, S. Suzuki, H. Shiromaru, Y. Miyake, K. Saito, I. Ikemoto, M. Kainosho, and Y. Achiba, Nature 357, 142 (1992).

[35] Y. Achiba, K. Kikuchi, Y. Aihara, T. Wakabayashi, Y. Miyake, and M. Kainosho, Mater. Res. Soc. Symp. Proc. 359, 3 (1995).

[36] N. Troullier and J. L. Martins, Phys. Rev. B 46, 1754 (1992). 